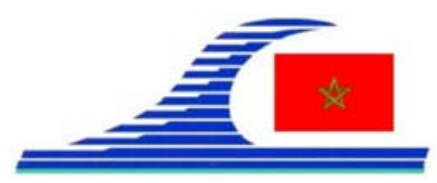

Conférence Méditerranéenne Côtière et Maritime EDITION 2, TANGER, MAROC (2011)

Coastal and Maritime Mediterranean Conference

Disponible en ligne - http://www.paralia.fr - Available online

\title{
Modélisation marine : l'impact des données atmosphériques COADS sur la réponse du modèle océanique régional ROMS sur le Sud du Maroc
}

\author{
Abderrahim MOUJANE ${ }^{1,2}$, Mohamed CHAGDALI ${ }^{2}$, Soumia MORDANE ${ }^{2}$ \\ 1. Direction de la Météorologie Nationale, Casablanca, Maroc. \\ abderrahim.moujane@gmail.com \\ 2. Faculté des Sciences Ben M'Sick, Casablanca, Maroc. \\ fest_20@yahoo.fr;mordanesoumia@yahoo.fr
}

\section{Résumé :}

L'intervention du courant des Canaries dans le domaine du sud du Maroc a un effet incontournable sur la SST (Sea Surface Temperature) à l'offshore, à la côte et sur l'upwelling des Canaries (IRD, site web).

Le modèle ROMS (Regional Ocean Modeling System) forcé par des données climatologiques COADS (Comprehensive Ocean-Atmosphere Data Set) reproduit bien la dynamique de cette région d'upwelling.

Simultanément, cette étude montre que la présence des îles Canaries perturbe le courant des Canaries et génère de l'activité méso-échelle.

Enfin, entre les zones côtières et océan ouvert le contraste de la température de la surface marine répond aux fluctuations du vent du large à la côte et à l'impact du climat dynamique global.

\section{Mots-clés :}

ROMS - COADS - Forçage - Upwelling - méso échelle - Courant

\section{Introduction}

L'importance du forçage du vent dans la circulation océanique a été largement étudiée. En commençant par les études d'Ekman en 1902, le stress de vent est reconnu comme le plus important producteur de l'upwelling côtier depuis Smith en 1968 (ENRIQUEZ \& FRIEHE, 1995).

Des études récentes ont montré la sensibilité des courants océaniques aux structures de vent de méso échelle (CAPET et al., 2004). La structure spatiale du vent côtier et des flux de chaleur peut avoir une grande influence sur les schémas de la circulation d'upwelling. La circulation atmosphérique au large des côtes est modifiée par la topographie côtière des continents qui génère une variabilité à petite échelle dans les structures de vent (CHAO et al., 2003).

L'objectif de ce travail est d'étudier la réponse du modèle océanique ROMS aux forçages dynamiques des données climatologiques mensuelles issus de COADS sur le domaine du Sud du Maroc. 
La connaissance de la Mer :

un vecteur du développement durable en Méditerranée

\section{Matériels et méthodes}

\subsection{Jeu des données COADS}

COADS est le fruit de la cooperation entre le NSF/NCAR (National Science Foundation of the National Center for Atmospheric Research) et la NOAA (National Oceanic and Atmospheric Administration). COADS est une base de données mondiale des observations de la surface de la mer de 1784 à 1997.

Les données COADS sont prises principalement par les navires (marchand, la recherche océanique, de la pêche, de la marine, etc) et de bouées dérivantes et ancrées (CLAUDE \& MENDELSSOHN, 1998). Les données utilisées sont le vent et les flux de chaleur construit à partir des données climatologiques mensuelles issus de COADS de $0.5^{\circ}$ de résolution.

\subsection{Simulation ROMS}

Les simulations réalisées par le modèle hydrodynamique ROMS ont une résolution spatiale de $7-8 \mathrm{~km}\left(1 / 12^{\circ}\right)$. Trente niveaux verticaux ont été choisis avec un paramètre $\theta$ s d'étirement de surface de 7 , un paramètre $\theta b$ d'étirement au fond de 0 et un minimum de profondeur de $10 \mathrm{~m}$.

Le nombre de points de calcul de la grille tridimensionnelle du modèle est, dans cette configuration, $155 \times 134 \times 30$, soit 623100 points.

La bathymétrie de notre domaine est issue du produit GEBCO (General Bathymetric Chart of the Oceans) de résolution $1 \mathrm{~km}$. Celle-ci a été lissée pour limiter les erreurs dues au traitement du gradient de pression en coordonnées sigma (SHCHEPETKIN \& MCWILLIAMS, 2003).

Le forçage par le vent et les flux de chaleur est construit à partir des données COADS. Les conditions initiales pour la température $(\mathrm{T})$ et la salinité $(\mathrm{S})$ proviennent des données climatologiques mensuelles WAO dont la résolution est de $0.5^{\circ}$. Après constructions des conditions aux limites, nous avons procédé à une simulation de 3 ans afin de calibrer le modèle océanique "spin up". Puis nous avons fait une simulation d'une année pour avoir un cycle annuel.

\section{Résultats : analyse des températures de la surface de la mer}

Pour analyser les résultats du modèle, nous avons choisi les simulations de ROMS sur la période d'une année. Des moyennes saisonnières sont produites et présentés ici.

Les Figures ci-dessous montrent que la répartition des températures de surface allant des hautes valeurs au large vers les basses valeurs à la côte, mettant en évidence le rôle du forçage sur le développement de l'upwelling côtier. Une saisonnalité beaucoup plus marquée de la température de surface de la mer au large (gyre subtropical) qu'à la côte. On note toutefois des eaux côtières plus froides dans la saison d'hiver et du printemps. Aussi, il est notable la présence de structures de type filament. 
A best knowledge of the Sea: A sustainable development vector in Mediterranean
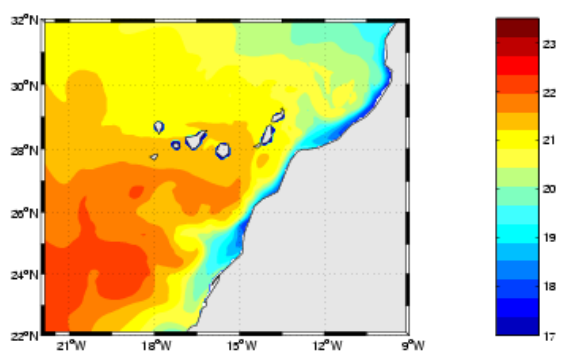

Figure 1. Température de surface pour la saison d'automne (octobre, novembre et décembre).
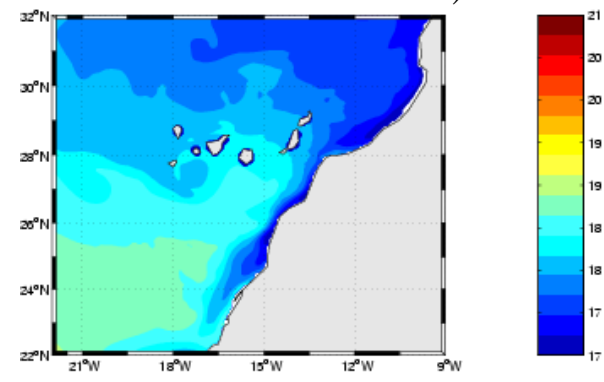

Figure 3. Température de surface pour la saison du printemps (avril, mai et juin).
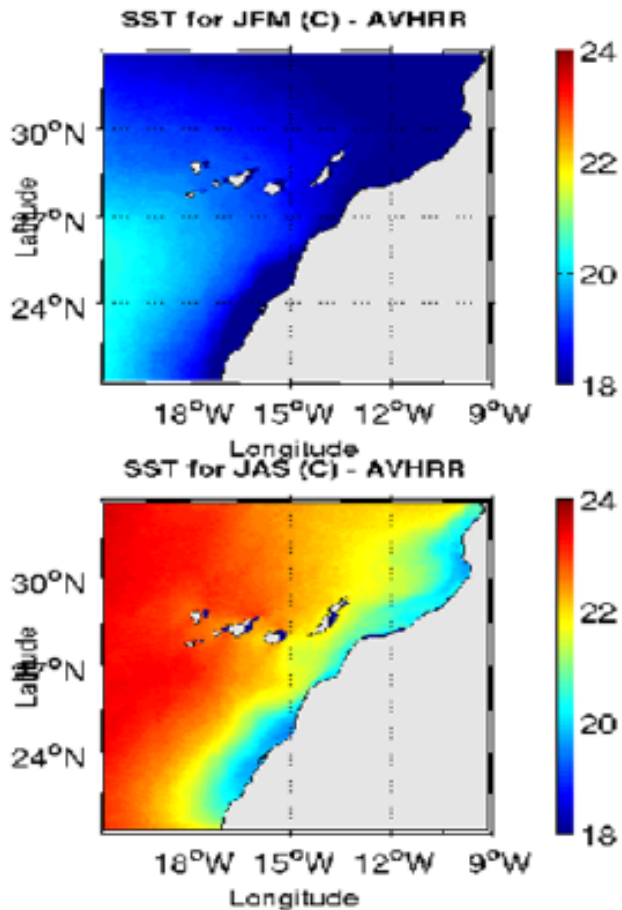
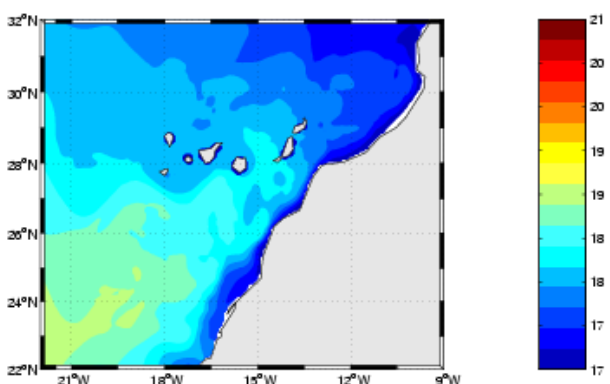

Figure 2. Température de surface pour la saison d'hiver (janvier, février et mars).
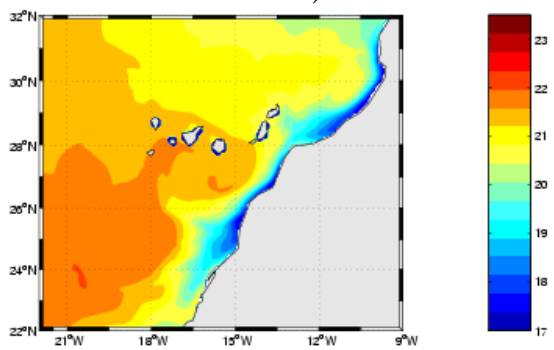

Figure 4. Température de surface pour la saison de l'été (juillet, août et septembre).

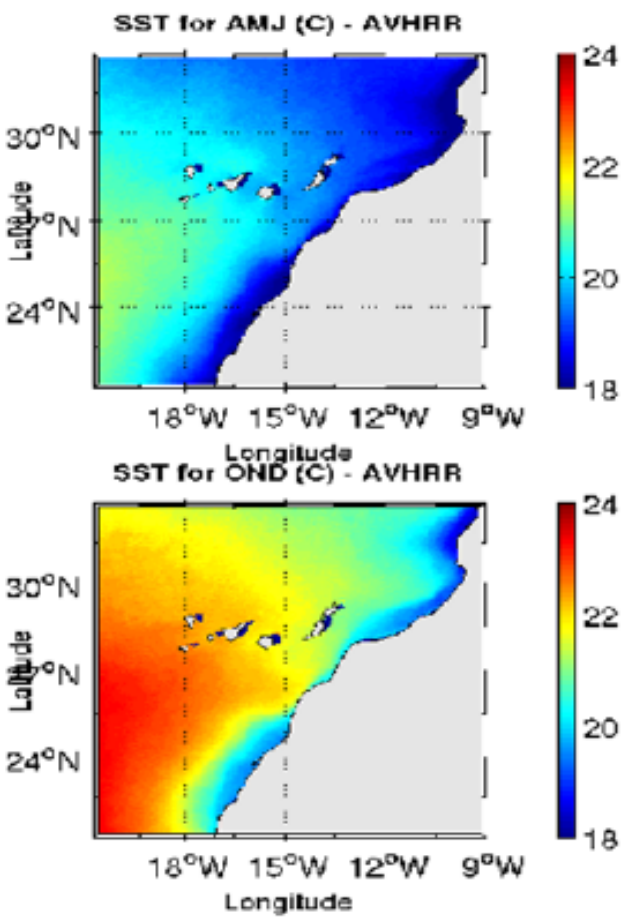

Figure 5. La température de la surface de la mer AVHRR. 
La connaissance de la Mer :

un vecteur du développement durable en Méditerranée

\section{Validation}

Les données de validation utilisées sont celles de l'AVHRR (Advanced Very High Resolution Radiometer) issues du satellite NOAA.

Les graphes de la figure 5 présentent la SST saisonnière des données AVHRR, ces cartes révèlent une harmonie considérable avec la SST des simulations du modèle ROMS dans la Figure 3 et 4.

\section{Conclusion}

En effet, dans cette configuration de référence, le modèle ROMS forcé par des données climatologiques COADS à faible résolution spatio-temporelle reproduit bien la dynamique de cette région d'upwelling. Simultanément, cette étude montre que la présence des îles Canaries perturbe le courant des canaries et génère de l'activité méso échelle. Cette activité est également associée aux principaux caps de la côte marocaine et généralement résultent de l'influence de la topographie côtière, de la géométrie du littoral, et des conditions atmosphériques synoptiques.

Alors, l'étude des structures fines du modèle atmosphérique (principalement vents et flux de chaleur) est susceptible d'apporter des informations sur le rôle que ces structures jouent sur la circulation océanique de cette région.

\section{Références bibliographiques}

CAPET X.J., MARCHESIELLO P., MCWILLIAMS J.C. (2004). Upwelling response to coastal wind profiles. Geophysical Research Letters, Vol. 31, n ${ }^{\circ}$ 13, L13311. doi:10.1029/2004GL020123

CHAO Y., LI Z., KINDLE J.C., PADUAN J.D., CHAVEZ F.P. (2003). A HighResolution Surface Vector Wind Product for Coastal Oceans: Blending Satellite Scatterometer Measurements with Regional Mesoscale Atmospheric Model Simulations. Geophysical Research Letters, 30(1), 1013. doi:10.1029/2002GL015729

CLAUDE R., MENDELSSOHN R. (1998). The development and the use of a climatic database for CEOS using the COADS dataset.

ENRIQUEZ A.G., FRIEHE C.A. (1995). Effects of wind stress and wind stress curl variability on coastal upwelling. Journal of Physical Oceanography, Vol. 25, $\mathrm{n}^{\circ} 7$, pp 1651-1671. doi:10.1175/1520-0485(1995)025<1651:EOWSAW>2.0.CO;2

IRD ( site web). Le courant des Canaries. Les dossiers thématiques de l'IRD, SUDS en ligne Les écosystèmes marins tropicaux. Les grands écosystèmes mondiaux d'upwelling [URL http://www.mpl.ird.fr/suds-en-ligne/ecosys/upwelling/canaries.htm ]

SHCHEPETKIN A.F., MCWILLIAMS J.C. (2003). A method for computing horizontal pressure-gradient force in an oceanic model with a nonaligned vertical coordinate, Journal of Geophysical Research, 108 (C3), 3090. doi:10.1029/2001JC001047 